\title{
La innovación educativa en el contexto de la Educación Superior Técnico-Profesional
}

\author{
Claudio Maregatti Solano \\ Orcid: 0000-0002-1285-501X \\ cmaregatti@inacap.cl \\ Universidad Tecnológica de Chile INACAP \\ María Luisa Arancibia Muñoz \\ Orcid: 0000-0002-1266-159X \\ marancibiam@inacap.cl \\ Universidad Tecnológica de Chile INACAP \\ Rosa Eliana Romero Alonso \\ Orcid: 0000-0003-2800-5092 \\ reromero@inacap.cl \\ Universidad Tecnológica de Chile INACAP
}

\section{Resumen}

La transformación de la Educación Superior Técnico-Profesional (ESTP) implica potenciar el desarrollo académico y contribuir al conocimiento en este ámbito. En este escenario, la generación de un Congreso Educativo se convierte en un espacio de reflexión donde se ponen en juego los recursos y capacidades académicas de los docentes que conforman distintos espacios académicos en Educación Técnico-Profesional. En este espacio confluyen conocimientos en torno a la innovación y la investigación educativa asociadas a temáticas como la problemática de las políticas educativas, modelos educativos, diseños curriculares, didácticos y pedagógicos en ESTP. En este artículo se presentan las reflexiones y consideraciones para la evaluación de las comunicaciones más representativas expuestas en un congreso de innovación educativa en contextos inclusivos de Educación Superior que se convirtieron, finalmente, en los capítulos de este libro con Referato. 
Palabras clave: Educación Superior Técnico-Profesional, innovación educativa, educación superior inclusiva.

\begin{abstract}
Higher Technical Education (HTE) transformation implies strengthening academic development and contributing to knowledge in this field. In this scenario, an educational conference becomes a space for reflection where academic resources and capabilities of the teachers who make up different academic spaces in Technical-Professional Education are put into play. In this space, knowledge converges around innovation and educational research associated with topics such as the challenges and opportunities of educational policies, educational models, curricular, and pedagogical designs in HTE. This article presents the reflections and considerations for the evaluation of a selection of communications presented at a conference on educational innovation in inclusive Higher Education contexts, which finally became the chapters of this peer-reviewed edited book.
\end{abstract}

Keywords: Higher Technical Education, educational innovation, inclusive Higher Education.

\title{
1. Introducción
}

La Educación Superior Técnico-Profesional chilena se encuentra en un proceso de profundos cambios que viene dado por las políticas educativas conducentes a mejorar los procesos académicos, la formación de nuevos profesionales y el aporte de estos conocimientos a la sociedad.

El objetivo del presente artículo es mostrar un análisis de los procedimientos y reflexiones en torno a las características del contexto, actores y prácticas innovadoras, así como los principales productos académicos asociados a la gestión de la innovación e investigación educativa desarrolladas en el congreso educativo de un contexto de Educación Superior Técnico-Profesional (ESTP).

Desde la política educativa se provee el marco regulatorio para caracterizar las instituciones innovadora en la ESTP (Decreto Exento núm. 848-2016), junto con la nueva Ley de Educación Superior Chilena (Ley 21091, 2018), que incorpora las funciones académicas de docencia, vinculación con el medio, innovación y, 
en cierta medida, investigación para los niveles de Centros de Formación Técnica e Institutos Profesionales, con el objetivo de aportar a la calidad de los procesos de enseñanza-aprendizaje y con nuevos conocimientos de especialidades al mundo productivo.

Este nuevo escenario busca responder a los complejos, acelerados y cambiantes fenómenos globales que afectan a los procesos sociales, productivos, educativos y académicos. Las instituciones que forman profesionales a través de su política y modelo educativo reorganizan sus recursos, saberes académicos y el mundo del trabajo en diferentes proyectos conducentes a involucrar y sensibilizar a los actores educativos técnicos profesionales, que en la mayoría de los casos significa transformar una institución de educación superior docente a una académica.

En este sentido, desde el Centro de Innovación en Educación CIEDU de la Universidad Tecnológica de Chile, INACAP, han surgido diferentes iniciativas innovadoras, como el Concurso de Innovación Docente (CID), la gestión, coordinación y dictación del Magíster en Pedagogía Aplicada a la Educación Superior y el Congreso Educativo, que han tenido como objetivo precisamente gestionar la innovación, en distintos niveles experiencias, buenas prácticas e investigaciones en el ámbito educativo, convirtiéndose cada uno de ellos en espacios de interacción académica en torno a las innovaciones educativa (Saez, Soto, Flores, Neira y Maregatti, 2018), cuyo propósito es generar las condiciones para la reflexión docente y la adopción de buenas prácticas que impacten en su quehacer académico y en la calidad de la formación de nuevos profesionales.

La evolución de dichos espacios de innovaciones ha intentado adaptarse a las profundas transformaciones del sistema educativo poniendo en evidencia los límites y alcances de la innovación educativa vinculadas a la sensibilización docente, su formación, interacción entre pares, la difusión, publicación, el escalamiento y la transferencia educativa, y como resultado se han obtenido nuevas formas de interacción para la producción académica. 


\section{La transformación de las instituciones de Educación Superior Técnico-Profesional}

La transformación de las instituciones que responden a las demandas de un mundo globalizado implica un periodo de transición del paso de una institución docente a una académica que afecta tanto a la unidad educativa en su conjunto como a sus distintos actores. Esta nueva forma de percibir la acción educativa afecta principalmente a los docentes en su quehacer cotidiano, tanto en los procesos de enseñanza-aprendizaje como en su propio proceso formativo.

En el nuevo contexto académico, la gestión del conocimiento exige estar acorde a los ritmos y tiempos de la producción y reproducción del conocimiento. La lógica global de la producción académica dentro de los sistemas educativos implica la generación de las condiciones para la circulación de la información y el conocimiento en sus distintos niveles: reflexiones prácticas, experiencias, innovaciones, transferencias e investigaciones educativas. Esto significa ordenar y organizar el saber docente institucional para mejorar la calidad de los aprendizajes a través de la formación docente y académica.

La incorporación de las funciones académicas de docencia, vinculación con el medio, innovación e investigación es un proceso paulatino, y ha de tener como punto de partida la tradición docente de la institución y, fundamentalmente, lograr su equilibrio con la función investigativa (Bolivar, 2017).

Dicha condición tiene que estar dada desde la Política Institucional, la cual ha de promover una cultura organizacional colaborativa y participativa en torno a la innovación, transferencia e investigación educativa y productiva. Esta ha de plasmarse en el Modelo Educativo Institucional de tal modo que oriente la práctica académica y principalmente debe expresarse en el modelo de Diseño Curricular, que lleva concretamente el accionar académico al aula.

Específicamente, los diseños didácticos y pedagógicos deben tributar a las diversas actividades que son propias de las funciones académicas, todo esto de manera intencionada y progresiva. Es aquí donde la innovación educativa se presenta como el modo deliberado con el cual se operacionaliza el quehacer educativo de los diferentes actores involucrados en la acción educativa, en especial, para el docente. 
De ahí la importancia de identificar la historia del conocimiento y saber docente de la institución, que se ha traducido en el aula a través de desarrollo de las estrategias didácticas que, al ser sistematizados por la función investigativa, articula nuevos saberes que pueden ser canalizados en espacios de producción académica como un congreso educativo.

En la práctica, se tienen que generar espacios académicos en los cuales se difundan y publiquen las principales reflexiones y experiencias educativas de los docentes. Son instancias que invitan a sistematizar las buenas prácticas y la creatividad de estos, a través de las innovaciones, junto con los procesos investigativos vinculados a ellas.

\section{De las prácticas docentes al saber académico}

La naturaleza de la gestión de la innovación es reunir el saber docente para ser redistribuido. Sin embargo, tiene que adaptarse de manera flexible a los diferentes escenarios y cambios del contexto global, social, institucional, curricular, didáctico y pedagógico. Esta misma característica, en su desarrollo, permite canalizar el saber docente hacia un saber académico, entendido como un proceso progresivo de transformación en la forma de percibir los fenómenos educativos desde las prácticas docentes.

Disponer de recursos tecnológicos y el uso de estos es fundamental. Por una parte, promover el uso de tecnología en el cuerpo docente incentiva a crear nuevas estrategias didácticas para la utilización de las tecnologías. Por otra, permite contar con espacios virtuales que contengan las principales experiencias e innovaciones educativas para su difusión y escalamiento, además de proporcionar espacios de interacción docente.

La transformación del saber docente al saber académico tiene en la formación docente una de sus características esenciales. En este sentido, el proceso de mentoría (Fish, 2017) entre pares permite a los docentes más experimentados en innovación e investigación explicar los procedimientos a los docentes nóveles y, a la vez, llevar estas prácticas al aula. En esta interacción, surgen los primeros insumos para las reflexiones en torno a la innovación educativa, los recursos tecnológicos y metodologías utilizadas, la investigación asociada, la formulación de proyectos, la produc- 
ción de artículos, las formas de sistematización de los saberes docentes y los procesos académicos en general.

Desarrollar las competencias y funciones académicas no está exento de dificultades, principalmente asociadas a los tiempos destinados para la formación docente a través de la mentoría, ya sea que los docentes mentores estén dispuestos a formar a otros docentes desde sus experiencias o que los docentes mentorizados respondan a las limitaciones propias de las innovaciones educativa en relación con la percepción de los otros actores (Marcelo, 2016) y el contexto en que se desarrollan.

Para reducir las brechas asociadas a los procesos académicos y de la innovación educativa, se puede partir por identificar los docentes de acuerdo a sus características académicas y profesionales. En sus prácticas cotidianas, algunos docentes muestran motivación y disposiciones naturales a las prácticas académicas. Sistematizar el trabajo con ellos llevaría a diseñar un procedimiento de la interacción entre los docentes y académicos y la forma como estos se relacionan con los recursos tecnológicos y las innovaciones disponibles. Pero, además, permite estructurar los procedimientos para la incorporación de nuevos docentes a la práctica académica.

En la acción académica, implica que los docentes experimentados utilicen, por ejemplo, repositorios virtuales para promover el trabajo colaborativo y multidisciplinar en torno a temas y problemáticas comunes. Este punto de partida despliega la idea de la incorporación progresiva en el quehacer docente las funciones y la producción académica, cuyo resultado tiene que traducirse en la presentación de ponencias en congresos, formulación de nuevos proyectos de innovación educativa y elaboración de artículos académicos.

En consecuencia, se generan las condiciones y la sensibilización de los actores académicos para identificar las características de los proyectos innovadores que se implementan y, sobre todo, estructurar comunidades profesionales de aprendizaje (Bolivar, 2017) en torno a la innovación educativa como equipos interdisciplinares.

El trabajo colaborativo y la conformación de comunidades críticas y participativas son características propias de la investigación-acción (Bisquerra, 2012), donde surgen investigaciones en torno a diseños didácticos innovadores que han sido implementados. 
Este contexto académico permite la evaluación de resultados de proyectos innovadores donde el nivel de rigurosidad investigativa y didáctica, al incorporar el uso de tecnología en las actividades, permite conjugar las funciones académicas para contribuir a tratar temáticas y problemáticas propias de la Educación Superior Técnico-Profesional de hoy, como son: la reducción de brechas entre el perfil de ingreso de los estudiantes y el perfil profesional de la especialidad, la formación docente, los procesos de mentorías, la inclusión educativa, el desarrollo de estrategias activas y la evaluación de competencias. Poniendo en la acción académica los conceptos de innovación, vinculación con el medio, investigación y transferencia educativa, tipos de transferencia y la evaluación de la transferencia (Cano, 2015) como ejemlos para contrastar y validar ante la comunidad de actores académicos la productividad académica.

\section{El aporte al conocimiento científico sobre la Educación Superior Técnico-Profesional}

Un congreso educativo es la oportunidad de estructurar un espacio, donde, a modo de síntesis, confluyan experiencias de innovación, buenas prácticas y producción académica como consecuencia de la sistematización de los saberes educativos y profesionales de diferentes actores vinculados a la Educación Superior Técnico-Profesional.

Se aprecia en la experiencia de congresos internacionales una tendencia similar:

Con frecuencia dichos efectos se han considerado en términos de mejora del aprendizaje de los estudiantes y/o de su satisfacción, de la actividad de los docentes, de los procesos de cambio impulsados o de optimización de la organización, de los procesos y de los recursos empleados (...) puesto énfasis en la necesidad de seguir innovando, experimentando e investigando con el objetivo de mejorar los logros y, a la vez, difundirlos y transferirlos. (EDUTEC, 2018: 5)

Asimismo, un congreso educativo permite revisar las nuevas tendencias e innovaciones que están cambiando la educación a nivel mundial, identificando los recursos para mejorar la acción 
educativa, compartir, colaborar y construir espacios en torno a temáticas y problemáticas comunes (Congreso Internacional de Innovación Educativa, 2018).

Precisamente, el propósito del presente libro y de este artículo es mostrar las comunicaciones más representantes y destacadas que se presentaron en el VII Congreso Educativo de INACAP el año 2019. El congreso es una iniciativa del Centro de Innovación en Educación CIEDU, que tiene como misión:

(...) desarrollar y promover la innovación en la docencia para la mejora permanente de la calidad de la experiencia de aprendizaje de los estudiantes, apoyando el proyecto institucional con contribuciones a la formación de nuestros académicos y la apropiación de estrategias y tecnologías concebidas como oportunidades de desarrollo de conocimiento. (CIEDU, 2019)

Este año el congreso tuvo dos hitos importantes, que dieron la oportunidad de enriquecer con nuevos saberes, experiencias y recursos su realización. Por una parte, se realizó en conjunto con la Escuela de Humanidades y Educación de INACAP y, por otra, se abrió por primera vez a la comunidad académica nacional e internacional, de modo que se recibieron comunicaciones derivadas de investigaciones y de innovaciones educativas.

Desde sus inicios, el Congreso Educativo en sus versiones anteriores se ha convertido en un espacio donde diferentes actores educativos difunden y comparten experiencias innovadoras a la comunidad educativa, además de ser un foro de motivación para la adopción de nuevos conocimientos y prácticas exitosas para la promoción de una cultura innovadora en la Educación Superior.

Este importante evento educativo ha encarnado la misión, valores y principios que INACAP ha promovido a lo largo de sus 53 años de historia, liderando procesos conducentes a mejorar la calidad de la enseñanza y el aprendizaje de los estudiantes. Esta intención ha sido plasmada dentro de la política institucional y su modelo educativo, que busca responder a nivel organizacional a los profundo y acelerados cambios globales, sociales y educativos específicamente a nivel de la ESTP.

En este sentido, el séptimo congreso educativo se constituyó en un hito significativo a nivel institucional y nacional. Lo que compartimos en esta oportunidad fue producto de la historia y 
la madurez alcanzada como institución y, especialmente, como Centro de Innovación en Educación de INACAP que exigió movilizar sus capacidades para desplegar el saber acumulado antes durante y después del evento.

El mundo académico fue invitado a compartir experiencias y conocimientos con temas y problemas que forman parte del contexto de transformación actual. Por ello, este año el tema central del Congreso fue la "Construcción de una cultura innovadora para la inclusión en la Educación Superior Técnico-Profesional». En efecto, la educación técnico-profesional en nuestro país se ha caracterizado principalmente por desarrollar solo la función académica docente y, en menor medida, las funciones de innovación y la vinculación con el medio.

Como parte de la producción académica de excelencia, y a fin de consolidar el riguroso trabajo realizado, se decidió hacer este libro con referato externo seleccionando las mejores comunicaciones, después de un exhaustivo proceso evaluativo. Se recibieron 15 artículos, de los cuales quedaron solo los que presentamos en este libro, en los cuales se abordan temas como: el perfil de los estudiantes que ingresan a la Educación Superior técnico-profesional y las características de sus trayectorias académicas, los problemas de deserción y exclusión, el perfil de docentes, el trabajo de mentoría en la formación docente, las estrategias didáctica activas como la clase invertida, la evaluación de la innovación y los programas de inclusión en contextos de la Educación Superior.

A modo de resumen, los principales temas asociados a la innovación educativa en contextos inclusivos de Educación Superior con los cuales el lector se encontrará en este libro son los que presentamos a continuación.

El segundo capítulo de este libro hace hincapié en la caracterización del estudiante técnico-profesional en relación con su historia y trayectoria académica, especialmente secundaria, y cómo ello influye en el ingreso y en la retención de la matrícula en la Educación Superior técnico-profesional en el contexto chileno. La literatura demuestra que existen factores que determinan el ingreso de estudiantes secundarios a los estudios superiores, entre los cuales destaca el nivel socioeconómico, las modalidades de estudio, la oferta de Educación Superior, las expectativas personales y familiares (Sepúlveda, 2013; Sepúlveda, 2016; Olve- 
ra, González, Rodríguez y Poblano, 2019). Sobre los resultados más destacados de este artículo, realizado en una institución de Educación Superior técnico-profesional de Chile y denominado «Caracterización de estudiantes de Educación Técnico-Profesional respecto a su Educación Secundaria: reflexiones para la Educación Superior», demuestran que el ingreso promedio de años de los estudiantes es de 22, son estudiantes que viene principalmente de la Educación Humanista-Científica y son estudiantes que no entran a la ESTP de manera inmediata.

La relevancia de la temática y las discusiones abren nuevas dimensiones de investigación no solo referida a la política pública, sino también en su impacto en los diseños curriculares, didácticos y pedagógicos de la Educación Superior Técnica, es decir, también a la innovación pedagógica través de un mayor protagonismo del estudiante en su proceso de formación y la utilización de enfoques de enseñanza centrados en el estudiante (Salas, 2005; Cueto, 2016). La trayectoria de cada estudiante, considerando su historia académica secundaria y superior, además de la elección del programa de estudios y sus expectativas, es un desafío que va de la mano con las iniciativas actuales de modernización de la educación técnico-profesional en Chile, donde unos de sus pilares son las trayectorias laborales y educativas articuladas como base para mejora la calidad de la educación técnico-profesional (Mineduc, 2016).

Uno de los mayores desafíos a que se enfrenta el docente del siglo xxi es «reflexionar, analizar e interpretar aspectos intelectuales y afectivos y, en función de ello, realizar su práctica docente» (Cañedo y Figueroa, 2013: 2). Por lo tanto, la concepción que tienen los docentes sobre la formación de los estudiantes debe ser integral, y han de destacar el rol de la comunicación y la dimensión afectiva como elementos clave en la enseñanza (Tobón, 2014; Parra, Tobón y López, 2015). En este contexto, las concepciones sobre el quehacer académico, en particular los modelos de liderazgo, como el transformacional (Hermosilla, Amutio, Costa y Páez, 2016) o el modelo de liderazgo delta (Jordan y Garay, 2009), desarrollados en el tercer capítulo, permiten entender cuáles son las características más significativas que un docente tiene que desarrollar en su práctica docente. Este artículo, titulado «Aportes hacia la construcción de un perfil docente para el primer año: la importancia del vínculo empático, el valor acadé- 
mico y el trabajo colaborativo", contribuye en la comprensión de un perfil docente, a partir de la percepción de este.

Los resultados del estudio coinciden en que la enseñanza debe darse en un contexto de exigencia, pero también de cercanía y confianza, lo que está en directa relación con los modelos educativos actuales (Flores, 2011). Destaca el vínculo empático y el trabajo colaborativo, al permitir acciones de liderazgos y de cooperación que favorecen la formación de sus estudiantes como de su propio desarrollo profesional (Ellenari, Gil y Fiorese, 2012).

En el cuarto capítulo se destaca una investigación aplicada basada en la implementación de mentorías a un grupo de docentes nóveles de una institución de Educación Superior sobre una iniciativa de investigación y desarrollo denominada «Activando resolución de problemas en el aula» (ARPA) (Felmer y PerdomoDíaz, 2017). El capítulo se denomina «Mentoría docente para instalar estrategias de resolución de problemas en matemáticas iniciales». La base teórica se situó en el concepto de mentoría transicional, fundamentada en una relación de coaprendices o colegas, en la que se valoran las diferencias y la complementariedad (Cuéllar, González y Espinosa, 2019).

Los resultados de la intervención reflejan mejores resultados en términos de participación y rendimiento en los cursos de los docentes participantes, además de la satisfacción con la práctica docente que favorece la enseñanza de las matemáticas, lo cual potencia específicamente la resolución de problemas.

El aprendizaje de contenidos complejos, teóricos y abstractos en el ámbito de las matemáticas es un fenómeno difícil para la enseñanza de estas. Se convierte en un desafío trabajar con el enfoque denominado flipped classroom, o enfoque invertido, que, de acuerdo a la literatura, ha demostrado que contribuye a mejorar la interacción entre el profesor y el estudiante, y entre estudiantes, lo que resulta beneficios para la enseñanza de la matemática, ya que se potencia la discusión en clase y un mejor aprendizaje entre pares (Jungic, Kaur, Mulholland y Xin, 2015). En el quinto capítulo se presenta el desarrollo de una innovación pedagógica basada en un modelo pedagógico activo de flipped classroom, el cual facilita el aprendizaje (Jensen, Kummer y Godoy, 2014). El título del capítulo, "Clase invertida para la formación inicial de educadoras diferenciales sobre aprendizaje matemático", incluye que este modelo pedagógico, logra mejores resultados de apren- 
dizaje. Entre los resultados destacan la alta valoración modelo pedagógico de clase invertida y el trabajo en equipo.

En la década de los noventa, el Informe Delors llamado «La educación encierra un tesoro», de la Unesco, amplía, de manera significativa, la misión de las organizaciones educativas para el siglo XxI funciones, además de formar capital humano para participar del mercado y la industria mundial, se encuentran el desarrollo personal, la formación de valores democráticos, la función de movilidad social de la educación, el pensamiento crítico, el uso de tecnologías de comunicación e información, la coexistencia pacífica, hacer retroceder la pobreza, la función de garantizar la igualdad de oportunidades, entre otros (Delors, 1996). Esto pone en la palestra un nuevo foco de la innovación que se observará con intensidad en las políticas educativas de las últimas tres décadas.

Bajo este contexto, la innovación se convierte es una competencia clave para formar profesionales competentes en un mundo globalizado, complejo y dinámico (Navarro, Vaccarezza, González y Catalán, 2015). El sexto capítulo, «¿Cómo medir el aprendizaje en innovación? Análisis factorial confirmatorio del Innovator's Behavior Questionnaire (i)BQ en universitarios chilenos», muestra la validación de un cuestionario basado en Dyer, Gregersen y Christensen (2009), denominado (i)BQ (Innovator's Behavior Questionnaire), que mide el desarrollo percibido de la competencia en innovación. Los resultados estadísticos evidencian resultados similares obtenidos por Lledó, Maluenda, Varas y Zúñiga (2017) a partir del análisis factorial exploratorio realizado, y basados en la propuesta original de Dyer, Gregersen y Christensen (2009). Este estudio pretende contribuir a los indicadores que miden la competencia en innovación como parte del desarrollo de la innovación específicamente educativa en Chile.

El séptimo capítulo, «Programa de inclusión para actores de Educación Superior técnico-profesional», pretende aportar a la generación de una cultura inclusiva, entendiendo que la inclusión educativa surge como consecuencia de los altos niveles de exclusión y desigualdades educativas que persisten en la gran mayoría de los sistemas educativos (Echeita y Duk, 2008). Este programa de inclusión identifica a los estudiantes con discapacidad y origina mecanismos de acompañamiento y acciones per- 
tinentes que involucra a todos los actores educativos de la institución investigada. Su marco de acción responde a la Ley 20.422 promulgada en Chile, que señala que «las instituciones de educación superior deberán contar con mecanismos que faciliten el acceso de las personas con discapacidad...». Actualmente, la temática de inclusión y diversidad es parte del Plan Estratégico Institucional 2018-2022, de la institución investigada y se ha incorporado también en el proyecto educativo. Se han implementado varias acciones, entre ellas: charlas y reuniones reflexivas con la comunidad educativa, enseñanza desde la diversidad potenciando la temática de la cultura inclusiva en asignatura transversales, ajuste de integración en el proceso de admisión, formación de los docentes desde una perspectiva inclusiva y superar las barreras arquitectónicas considerando los principios de accesibilidad y diseño universal.

Finalmente, esperamos que los temas abordados en este artículo y en cada capítulo de este libro sirvan al lector para abrir nuevos caminos y hacer frente a la complejidad del proceso formativo en el logro de los aprendizajes, competencias y los perfiles de egreso de la ESTP. En su contexto, la innovación educativa se transforma en una significativa instancia para promover la inclusión educativa en sus múltiples dimensiones. Ello promueve, además, la investigación y la producción académica en los distintos actores optimizando la formación de nuevos profesionales.

\section{Referencias bibliográficas}

Barber, M.; Mourshed, M. (2007). Cómo hicieron los sistemas educativos con mejor desempeño para alcanzar sus objetivos. PREAL.

Bisquerra, R. (2012). Metodología de la Investigación Educativa. La Muralla. Bolivar, A. (2017). «Docencia e investigación en la universidad: de una relación problemática a una productiva». Revista Gestión de la Innovación en Educación Superior, vol. 2: 11-33.

Cano, E. (2015). «Evaluación de la formación. Algunas lecciones aprendidas y algunos retos de futuro». Revista Educar, vol. 51(1): 109-125.

Cañedo, T.; Figueroa, A. (2013). «La práctica docente en educación superior: una mirada hacia su complejidad». Sinéctica, 41: 2-18. Recuperado el 4 de octubre de 2018 de: <http://www.scielo.org.mx/scielo.php?script=sci_arttext\&pid=S1665-109X2013000200004\&lng=es\&tlng=es>. 
Centro de Innovación en Educación (2019). INACAP/Centro de Innovación en la Educación/Quiénes Somos. Página Web. Recuperado el 28 de noviembre del 2019 de: <http://portales.inacap.cl/ciedu-inacap/quienes-somos/index>.

Congreso Internacional de Innovación Educativa (2018). Tecnológico de Monterrey. México. Recuperado el 28 de noviembre de: <http:// ciieanteriores.mx/2018/es/objetivos>.

Cuéllar Becerra, C.; González Vallejos, M. P.; Espinosa Aguirre, M. J.; Cheung, R. (2019). "Buen mentor" y "buena mentoría" según actores de programas de inducción a directores novatos chilenos». Psicoperspectivas, 18(2): 33-46. Recuperado de: <https://dx.doi. org/10.5027/psicoperspectivas-vol18-issue2-fulltext-1543>.

Cueto, S. (2016). Innovación y Calidad en Educación en América Latina. GRADE/ ILAIPP, Lima-Perú

Decreto Exento núm. 848/2016. Política Nacional de Formación Técnico-Profesional, Chile, 2016.

Delors, J. (1996). La Educación Encierra un Tesoro (Libro). Santillana.

Dyer, J. H.; Gregersen, H. B.; Christensen, C. M. (2009). «The Innovator' s DNA». Harvard Business Review, págs. 1-9.

Echeita, G.; Duk, C. (2008). «Inclusión educativa». Revista Iberoamericana sobre Calidad, Eficacia y Cambio en Educación, 6(2): 1-8. Recuperado de: <https://www.redalyc.org/pdf/551/55160201.pdf>.

EDUTEC (2018). EDUcación con TECnología : un compromiso social. Aproximaciones desde la investigación y la innovación (editores F. Xavier Carrera Farran, Francisco Martínez Sánchez, Jordi L. Coiduras Rodríguez, Enric Brescó Baiges, Eduard Vaquero Tió). Lleida: Edicions de la Universitat de Lleida; Palma de Mallorca: Asociación EDUTEC.

Ellenari, P.; Gil, M.; Fiorese, L. (2012). «Un proceso de construcción participada del perfil docente en una red de Instituciones de Educación Superior de América Latina». Revista de Docencia Universitaria, vol. 10(2): 121-147.

Felmer, P.; Perdomo-Díaz, J. (2017). «Un programa de desarrollo profesional docente para un currículo de matemática centrado en las habilidades: la resolución de problemas como eje articulador». Educación matemática, 29(1): 201-217. DOI: <https://dx.doi.org/10.24844/ em2901.08>.

Fish, J. (2017). «Aportes de la mentoría universitaria a la creación y el mantenimiento de una organización de aprendizaje continuo». Revista Gestión de la Innovación en Educación Superior, vol. 2: 54-76. 
Flores, C. (2011) «Involucramiento del profesor universitario: Asignatura pendiente para las instituciones de educación superior». Universidades, 61(50): 79-88.

Gobierno de Chile (2010). Ley 20.422. Establece normas sobre igualdad de oportunidades e inclusión social de personas con discapacidad. Biblioteca del Congreso Nacional de Chile. Recuperado de: <https://www.leychile.cl/Navegar?idNorma=1010903\&idParte $=8869259$ \&idVersion $=2010-02-10>$.

Hermosilla, D.; Amutio, A.; Costa, S. D.; Páez, D. (2016). «El liderazgo transformacional en las organizaciones: variables mediadoras y consecuencias a largo plazo». Revista de Psicología del Trabajo y de las Organizaciones, 32(3): 135-143.

Jensen, J.; Kummer, T.; Godoy, P. (2014). «Improvements from a flipped classroom may simply be the fruits of active learning». Life Sciences Education, 14: 1-12

Jordan, R.; Garay, M. (2009). Liderazgo real de los fundamentos a la práctica. Pearson Educación.

Jungic, V.; Kaur, H.; Mulholland, J.; Xin, C. (2105). «On flipping the classroom in large first year calculus courses». International Journal of Mathematical Education in Science and Technology, 46(4): 508-520.

Ley 21.091 Sobre Educación Superior. Diario Oficial de la República de Chile, Santiago, Chile, 29 de mayo de 2018.

Lledó, P.; Maluenda, J.; Varas, M.; Zúñiga, M. (2017). «Efectos del programa GEARBOX sobre hábitos vinculados a la innovación en estudiantes de ingeniería». En: U. de Concepción (ed.). Educación Transversal y Responsabilidad Social. II Encuentro de Educación de Competencias Genéricas en Educación Superior. IX Encuentro Internacional de Educación para la Responsabilidad Social. 2017. Santiago.

Marcelo C. 2016. «La innovación en la universidad del Gatopardo al IPhone». Revista Gestión de la Innovación en Educación Superior, vol. 1. MINEDUC (2016). Bases para una Política de Formación en Educación Técnico-Profesional. Chile.

Navarro, G.; Vaccarezza, G.; González, M. G.; Catalán, R. (2015). Construcción de conocimiento en educación superior. Educación de competencias genéricas en la Universidad de Concepción, Chile (Navarro, G., ed.). Concepción: Universidad de Concepción.

Olvera, F.; González, A.; Rodríguez, R.; Poblano, E. (2019). «Factores que intervienen en la elección de una Institución de Educación Superior (IES): Una Revisión Sistemática de Literatura». Educateconciencia, 21(22): 17-34 
Parra, H.; Tobón, S.; López, J. (2015). «Docencia socioformativa y desempeño académico en la educación superior». Paradigma, 36(1): 4255. Recuperado de: <http://ve.scielo.org/scielo.php?script=sci_arttext\&pid=S1011-22512015000100004\&lng=es\&tlng=es>.

Saez, M.; Soto, M.; Flores, I.; Neira, E.; Maregatti, C. (2018). SABE: Una experiencia de innovación, transferencia e investigación educativa. En: Halal, C.; Arancibia, M.; Maregatti, C.; Riquelme, I.; Romero, R.; Segura, D.; Zamorano, R. (eds.). VI Congreso Educativo INACAP: Formación para la innovación, aprender para enseñar (pp.177-183). Santiago, Chile: RIL Editores.

Salas, W. (2005). «Formación por competencias en educación superior. Una aproximación conceptual a propósito del caso colombiano». Revista Iberoamericana de Educación, 36(9): 1-10.

Sepúlveda, L. (2013). «Más jóvenes en la educación superior, ¿mayores esperanzas de igualdad social?: una reflexión sobre las aspiraciones de futuro de los estudiantes secundarios chilenos». Cuaderno de Educación, 51: 1-9.

- (2016). «Trayectorias educativo-laborales de jóvenes estudiantes de educación técnica en Chile: ¿Tiene sentido un sistema de formación para el trabajo en la educación secundaria?». Páginas de Educación, 9 (2): 49-84.

Tobón, S. (2014). Proyectos formativos: teoría y práctica. México: Pearson. Universidad Tecnológica de Chile INACAP (2015). Modelo Educativo Institucional. Documentación Oficial.

- (2016). Plan de Desarrollo Estratégico. PDE 2020. Documentación Oficial. 
1. La innovación educativa en el contexto de la

Educación Superior Técnico-Profesional.

Claudio Maregatti Solano, María luisa Arancibia Muñoz, Rosa Eliana Romero Alonso

1. Introducción

2. La transformación de las instituciones de

Educación Superior Técnico-Profesional

3. De las prácticas docentes al saber académico

4. El aporte al conocimiento científico sobre la

Educación Superior Técnico-Profesional . . . . . . . . . 15

5. Referencias bibliográficas .................. . 21

2. Caracterización de estudiantes de Educación Técnico-Profesional respecto a su Educación Secundaria: reflexiones para la Educación Superior

Javier Torres-Vallejos, Juan Ignacio Venegas Muggli, Simón Mundaca Toledo, Juan Carlos Oyanedel Sepúlveda

1. Introducción . . . . . . . . . . . . . . . . . . . 26

2. Metodología ......................... . 30

2.1 Muestra . . . . . . . . . . . . . . . . . . . . . 30

2.2 Variables . . . . . . . . . . . . . . . . . . . . . 32

2.3 Tratamiento de bases de datos . . . . . . . . . . . . 32

2.4 Análisis de datos..................... 33

3. Resultados ........................... 33

3.1 Características educativas y académicas en

Educación Superior ..................... . 34

3.2 Características educativas y académicas del establecimiento secundario de egreso de Enseñanza Media . . . . . . . . . . . . . . . . . . . . 36

4. Discusión y conclusiones. . . . . . . . . . . . . . . 37

5. Referencias bibliográficas ................. . . 39 
3. Aportes hacia la construcción de un perfil docente para el primer año: la importancia del vínculo empático, el valor académico y el trabajo colaborativo

Carlos Alberto Acevedo Cossio

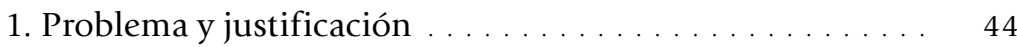

2. Marco teórico ....................... 45

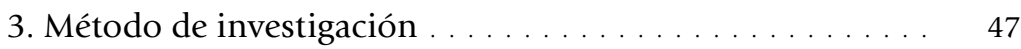

3.1 Diseño. . . . . . . . . . . . . . . . . . . 47

3.2 Participantes...................... 47

3.3 Técnicas e instrumentos de recolección de datos. . . . 48

3.4 Análisis de datos. . . . . . . . . . . . . . . . . . . . . 48

4. Resultados ... . . . . . . . . . . . . . . . . . . . . . . 49

4.1 Análisis de las entrevistas realizadas . . . . . . . . . 49

4.2 Análisis de cuestionarios . . . . . . . . . . . . . 50

4.2.1 Cuestionario de Estilo y Eficacia del Liderazgo . . 50

4.2.2 Cuestionario de Involucramiento Académico . . . 51

4.2.3 Índice de Reactividad Interpersonal . . . . . . . . . 52

5. Conclusiones y discusión ................. . 53

6. Referencias bibliográficas .................. 55

4. Mentoría docente para instalar estrategias de resolución de problemas en matemáticas iniciales

Cristian Iván Ramos Arrepol, Carlos Alberto Acevedo

Cossio

1. Problema y justificación . . . . . . . . . . . . . . . . 58

2. Marco teórico . . . . . . . . . . . . . . . . . . . . . . . 59

2.1 Trabajo colaborativo . . . . . . . . . . . . . . . . . 59

2.2 Mentorías ......................... 60

3. Método de investigación .................... 61

3.1 El perfil de entrada del docente . . . . . . . . . . . 62

3.2 Reclutamiento docente. . . . . . . . . . . . . 62

3.3 La caminata de aula . . . . . . . . . . . . . . . . . . 63

3.3.1 El primer paso . . . . . . . . . . . . . . . . 63

3.3 .2 Segunda ronda . . . . . . . . . . . . . . . . . . 64

3.3.3 Ronda final. . . . . . . . . . . . . . . . . . 64

3.4 Abordaje de situaciones críticas . . . . . . . . . . . . 64

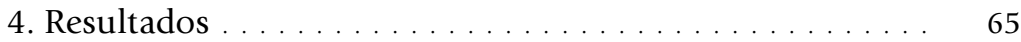

5. Conclusiones y discusión. . . . . . . . . . . . . . . . 69

6. Referencias bibliográficas ................... 70 
5. Clase invertida para la formación inicial de educadoras diferenciales sobre aprendizaje matemático

Laura Marjorie Espinoza Pastén

1. Problema y justificación . . . . . . . . . . . . . . . . . . 74

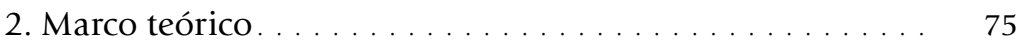

3. Proceso de la innovación. . . . . . . . . . . . . . . 76

3.1 Contexto ........................ 76

3.2 Diseño didáctico . . . . . . . . . . . . . . . 77

4. Resultados ......................... . 80

4.1 Resultados considerando el rendimiento académico. . 80

4.2 Resultados considerando la valoración de las estudiantes $\quad 81$

5. Discusión y conclusiones . . . . . . . . . . . . . . . 83

6. Referencias bibliográficas ................. 84

6. ¿Cómo medir el aprendizaje en innovación? Análisis factorial confirmatorio del Innovator's Behavior Questionnaire (i)BQ en universitarios chilenos. . . . . . 87 Jorge Maluenda Albornoz, Pedro Lledó Aninat

1. Problema y justificación . . . . . . . . . . . . . . . . . . . . . 89

2. Marco teórico . . . . . . . . . . . . . . . . . . . . . 90

3. Método............................. 92

4. Resultados ......................... 93

5. Conclusiones y discusión. . . . . . . . . . . . . . . . . 96

6. Referencias bibliográficas ................. 98

7. Programa de inclusión para actores de Educación Superior Técnico-Profesional . . . . . . . . . . . . . . . . 101

Marcela Pérez Poquet, Paulina Muñoz Villalobos, Victoria Aravena Rivas

1. Problema y justificación del estudio . . . . . . . . . . . . 102

2. Marco teórico . . . . . . . . . . . . . . . . . . . . . . 105

3. Metodología de investigación ................ 108

3.1 Descripción del contexto . . . . . . . . . . . . . . . 108

3.2 Participantes. . . . . . . . . . . . . . . . . . . . 109

3.3 Instrumentos y procedimientos . . . . . . . . . . . . . . 109

4. Resultados . . . . . . . . . . . . . . . . . . . . . . . 111

5. Conclusiones y discusión. . . . . . . . . . . . . . . . . . 113

6. Referencias bibliográficas ................. 113

Los autores. . . . . . . . . . . . . . . . . . . . . . . . . ${ }^{115}$ 